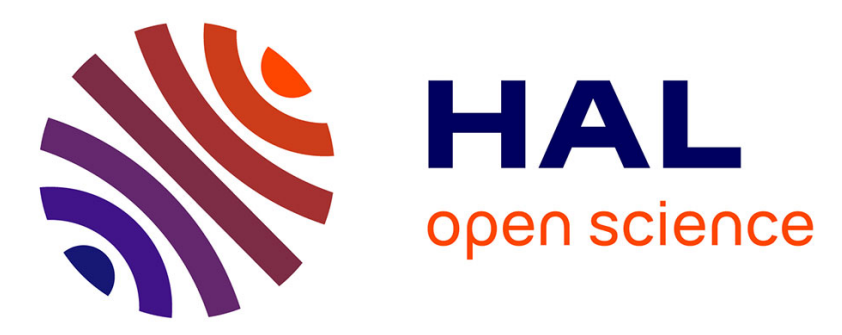

\title{
A Computer-Aided Process Planning Method Considering Production Scheduling
}

Eiji Morinaga, Hiroki Joko, Hidefumi Wakamatsu, Eiji Arai

\section{To cite this version:}

Eiji Morinaga, Hiroki Joko, Hidefumi Wakamatsu, Eiji Arai. A Computer-Aided Process Planning Method Considering Production Scheduling. IFIP International Conference on Advances in Production Management Systems (APMS), Sep 2015, Tokyo, Japan. pp.348-355, 10.1007/978-3-319-227566_43. hal-01417507

\author{
HAL Id: hal-01417507 \\ https://hal.science/hal-01417507
}

Submitted on 15 Dec 2016

HAL is a multi-disciplinary open access archive for the deposit and dissemination of scientific research documents, whether they are published or not. The documents may come from teaching and research institutions in France or abroad, or from public or private research centers.
L'archive ouverte pluridisciplinaire HAL, est destinée au dépôt et à la diffusion de documents scientifiques de niveau recherche, publiés ou non, émanant des établissements d'enseignement et de recherche français ou étrangers, des laboratoires publics ou privés. 


\title{
A Computer-Aided Process Planning Method Considering Production Scheduling
}

\author{
Eiji Morinaga ${ }^{1}$, Hiroki Joko ${ }^{1,2}$, Hidefumi Wakamatsu ${ }^{1}$, and Eiji Arai ${ }^{1}$ \\ ${ }^{1}$ Division of Materials and Manufacturing Science, Osaka University \\ ${ }^{2}$ Currently, Tokyo Institute of Technology \\ \{morinaga, hiroki.joko, wakamatu, arai\}@mapse.eng.osaka-u.ac.jp
}

\begin{abstract}
Process planning plays an important role as a bridge between product design and manufacturing. Computer-aided process planning (CAPP) has been actively discussed in this half century, and numerous works have been conducted. To meet recent strong requirements for realizing agile manufacturing, a set of flexible process planning methods have been developed. Those methods dealt with process planning only for one product and generate the optimal plan which achieves the shortest total machining time. However, in real manufacturing, multiple workpieces are machined with multiple machine tools in the same period, and pursuing the shortest machining time for each workpiece may result in poor productivity. This research aims to enhance those methods from the point of view of total productivity. Selection of the optimal process plan in the conventional methods and production scheduling were merged and then formulated as a 0-1 integer programming problem. This method was applied to a simple example and its potential was shown.
\end{abstract}

\section{Introduction}

Process planning deals with selection of necessary manufacturing processes and determination of the sequence in which they are applied to convert product design data into a real product. Since it plays an important role as a bridge between product design and manufacturing, computer-aided process planning (CAPP) has been a topic of discussion in this half century and numerous works have been conducted [1].

Due to recent diversified and changeable customers' needs, there is a need to realize agile manufacturing [2] that is capable of immediately adapting to changes in the manufacturing situation, that is, flexible manufacturing systems need to be realized. This need is also being addressed in research related to CAPP [3]. This research aimed to develop autonomous machine tools that require no NC programming and can flexibly adapt to changes in the manufacturing situation, and a flexible process planning method for rough milling was proposed. This method consists of four main steps-(i) decomposing the total removal volume (TRV) through the application of decomposition rules to transform it into sets of machining primitive shapes (MPSs), (ii) converting each of the MPS sets to a set of machining features (MFs) by determining a machining sequence for 
each set of MPSs and recognizing each MPS as an MF, (iii) executing rough operation planning by applying a tool selection rule and a case-based reasoning system for cutting condition decisions, and (iv) extracting the optimal set of MFs to achieve the shortest machining time with information on the machining sequence and the utilized tools as the optimal process plan. With this method, process plans are generated and then the optimal plan is selected. Therefore, when the manufacturing situation changes, it is possible to quickly provide a new optimal plan by executing steps (iii) and (iv). Several enhancements have been made to this method for enabling extraction of a better set of MFs [4], for taking multi-axis milling and reducing computational complexity [5], and for improving computational efficiency [6].

All of these methods deal with process planning to create one product using a single milling machine. However, in real manufacturing, multiple workpieces are machined with multiple machine tools in the same period, and pursuing the optimal plan without consideration of total production may result in poor productivity. This paper presents an enhanced method considering this point. The selection problem of the optimal process plan in the conventional methods and production scheduling problem are merged, and selection of a set of the optimal process plans from the productivity point of view is formulated as a $0-1$ integer programming problem.

This paper is organized as follows. The next section provides an outline of the conventional method [6]. Section 3 describes integration of the selection of the optimal process plans for multiple products and the production scheduling problem, and provides formulation of the integrated problem as a 0-1 integer programming problem. This method is applied to a simple example in Section 4 , and Section 5 presents our conclusion.

\section{Outline of Conventional Flexible CAPP Method}

This section provides an overview of the conventional method $[5,6]$, which will be improved considering production scheduling in Section 3. In this method, it is assumed that all the surfaces of a workpiece and the product are parallel to the $x y, y z$ or $z x$ plane of an orthogonal coordinate system and the tool approaches the workpiece along one of these axes, since this method is for rough machining. Process planning is performed by the following six steps:

\section{TRV extraction}

The total removal volume (TRV), which is defined as the volume to be eliminated from a workpiece to obtain the product shape, is calculated by subtracting the product shape from the workpiece shape (Fig. 2).

2. Concavity-based division

A cutting plane is generated at a concave part of the TRV contour, and then the TRV is divided by that plane. This process is iterated until cutting planes are generated for all concave parts, and the TRV is finally converted into a set of machining primitive shapes (MPSs). The type of the MPS set depends 
on the generation direction and sequence of the cutting planes. Therefore, multiple sets of MPSs can be produced from one TRV (Fig. 3).

3. Machining sequence and direction assignment

For each of the MPS sets, the machining direction (Fig. 1) for each MPS and the machining sequence for those MPSs are considered.

4. MF recognition

The machining directions and sequence assignment in the previous step makes it possible to regard each MPS as a machining feature (MF) based on the number of its "open faces" and the relationship between its vertices and edges. For each set of MPSs, multiple sets of MFs are generated depending on the direction and sequence (Fig. 4).

5. Process plan generation

For each of the MF sets, a tool to be used for each MF is selected from a given set of available tools by applying a given rule, and the machining condition for the MF is decided by a case-based reasoning system [7]. This operation generates, for each MPS set, sets of MFs including information about the machining sequence, the machining directions, and the tools to be used - that is, a process plans is generated.

6. Evaluation

For each process plan of each MPS set, the total machining time $\bar{T}$ is estimated using the following equations:

$$
\begin{aligned}
\bar{T} & :=\sum_{i=1}^{n} T_{i}+T_{\text {tool }} C_{\text {tool }}+T_{d i r} C_{d i r} \\
T_{i} & :=\frac{V_{i}}{D_{i a} D_{i r} F_{i}},
\end{aligned}
$$

where $T_{i}$ and $V_{i}$ stand for the machining time and the volume of MPS $i, D_{i a}$ and $D_{i r}$ are the depth of cut in the axial and radial directions of the tool used for the MPS, $F_{i}$ is the feed speed of the tool, $T_{\text {tool }}$ and $T_{d i r}$ are the times required for tool change and direction change, and $C_{\text {tool }}$ and $C_{d i r}$ are the number of tool changes and direction changes, respectively. The plan that achieves the shortest total machining time is selected as the optimal process plan for the MPS set by the full search [5] or mathematical optimization framework [6]. After performing this operation for all MPS sets, the estimated total machining times for the optimal plans are compared with each other. The optimal plan for which the estimated total machining time is the shortest is ultimately output as the optimal process plan for the TRV.

In real manufacturing, multiple workpieces are machined by using multiple machine tools in the same period generally. If this method is applied to those workpieces, the optimal plan is generated for each of them. However, the set of the optimal plans would not be truly optimal from the point of view of manufacturing systems, since multiple workpieces cannot be machined by a machine at the same time. Therefore, it is desirable to integrate process planning by this method with production scheduling. 


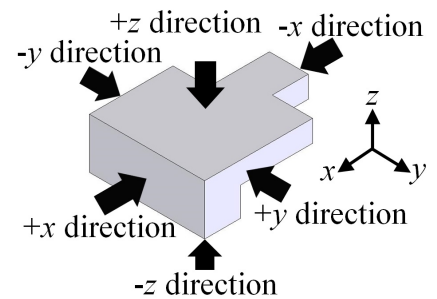

$-z$ direction

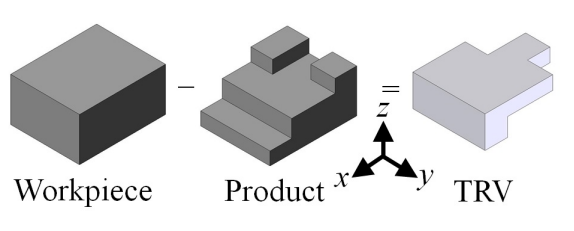

Fig. 2. TRV Extraction.

Fig. 1. Definition of machining directions.

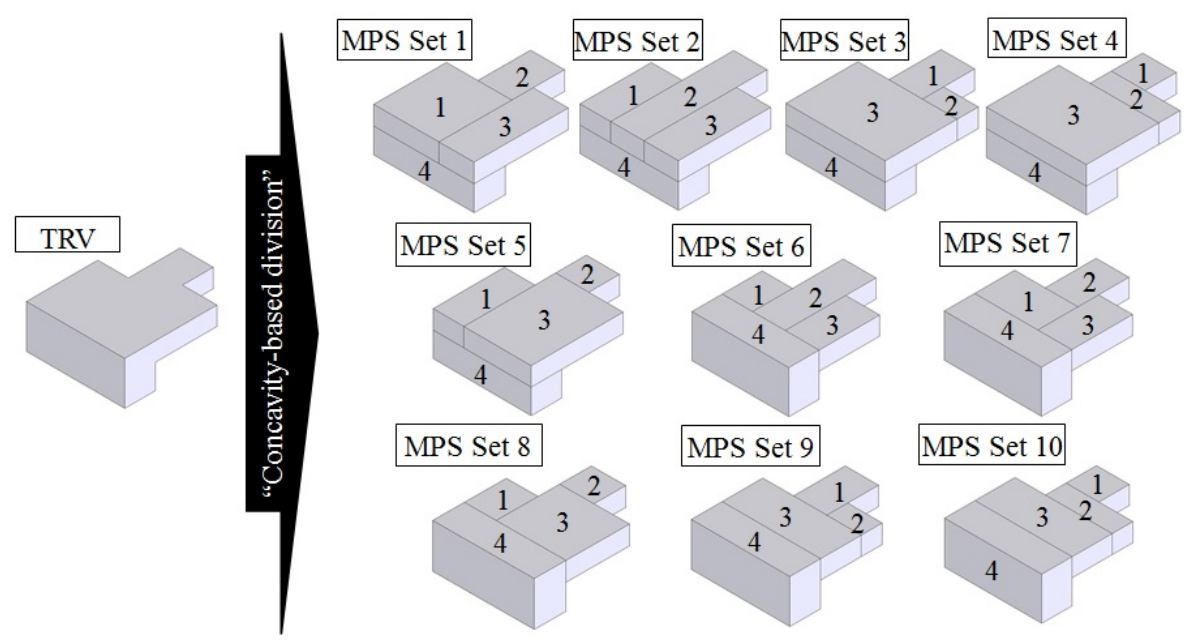

Fig. 3. Sets of MPSs extracted from the TRV. The numbers in each set are the identification numbers for the MPSs.
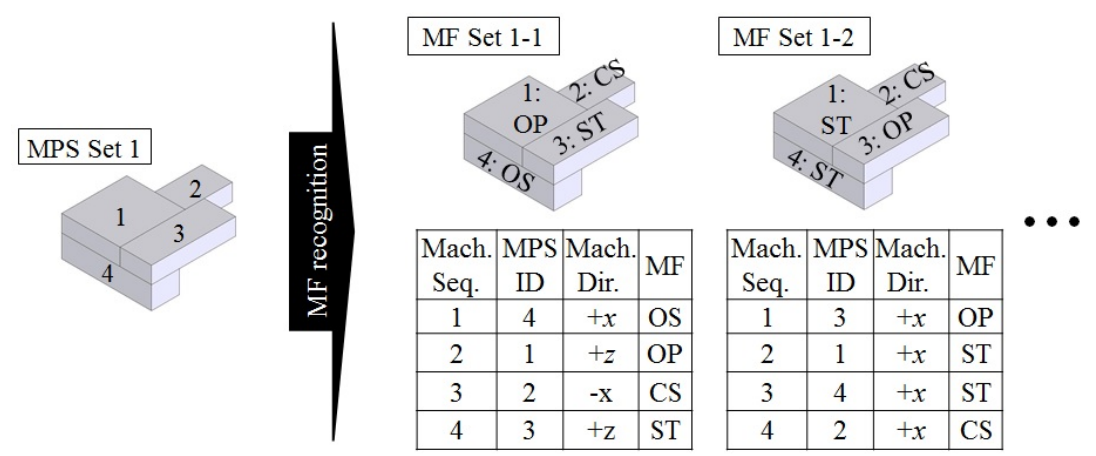

Fig. 4. MF recognition for an MPS set. Strings in each set stand for feature types ("CS", "OS", "OP", and "ST" stand for "closed slot", "open slot", "open pocket", and "step", respectively). 


\section{Flexible CAPP Method Considering Production Scheduling}

This section provides formulation of the integrated problem of the optimal plan selection and production scheduling as a $0-1$ integer programming problem. Two 0 -1 variables $x_{j v S_{n} \cdots S_{1} d t}$ and $z_{j p m \delta}$ are introduced, where $j \in \mathcal{J}:=\{1, \ldots, J\}$ and $v \in \mathcal{V}:=\{1, \ldots V\}$ stand for the ID number of product and MPS set of the product, respectively, and $n_{j}$ represents the total number of MPSs. $S_{k}$ is the ID number of the MPS that is machined after machining other $k-1$ MPSs, and $S_{1}$ takes its value in $\mathcal{N}_{j}:=\left\{1, \ldots, n_{j}\right\} . S_{k}, k \geq 2$ takes a value in $\{0\} \cup \mathcal{N}_{j}, k \geq 2$, and $S_{\tilde{k}}=0, \forall \tilde{k}>k$ means that only the first $k$ MPSs has been machined. $d \in\{1, \ldots 6\}$ stands for machining direction $(1:+x ; 2:-x ; \cdots 6:-z)$. $t \in \mathcal{T}:=\{1, \ldots T\}$ and $m \in \mathcal{M}:=\{1, \ldots, M\}$ are the ID number of the utilized tool and machine, respectively, $p \in \mathcal{N}_{j}$ is the process number, and $\delta \in\{1, \ldots, \Delta\}$ represents a period. $x_{j v 0 \cdots 0 S_{k} S_{k-1} \cdots S_{1} d t}=1$ means that the MPS $S_{k}$ of the MPS set $v$ for the product $j$ is machined in the direction $d$ with the tool $t$ after machining MPS $S_{1}, S_{2}, \ldots, S_{k-1}$ in this order. Similarly, $z_{j p m \delta}=1$ means that the machine $m$ deal with the process $p$ of the product $j$ at the period $\delta$. Let $\tau_{j p}$ is the required time for the process $p$ of the product $j$ and $T_{m}$ is the set of tools implemented on the machine $m$, then the problem of finding the optimal process plans for the products which achieve the smallest makespan can be described as a 0-1 integer programming problem by the following equations and inequalities (3)-(18). Equations (4)-(5) and inequalities (6)-(7) assure, for any product, that an MPS is always machined at each turn of the machining sequence and that an MPS is machined only once, respectively. Inequalities (8)-(10) and (11)(13) are for preventing invalid machining sequences. $P_{j v S_{n_{j}} \cdots S_{1} d t}$ which stands for the machining time of the MPS specified by $x_{j v S_{n_{j}} \cdots S_{1} d t}$ is calculated in advance and is set to a huge value if the machining is infeasible. Equation (14) including $P_{j v S_{n_{j}} \ldots S_{1} d t}$ merges the CAPP problem with the scheduling problem whose constraints are given by equation (15) and inequalities (16)-(18).

minimize: $\max _{j, p, m, \delta}\left\{\delta \cdot z_{j p m \delta}\right\}$

subject to:

$$
\begin{aligned}
& \sum_{v=1}^{V} \sum_{S_{1}=1}^{n} \sum_{d=1}^{6} \sum_{t=1}^{T} x_{j v 0 \cdots 0 S_{1} d t}=1, \forall j \in \mathcal{J} \\
& \vdots \\
& \sum_{v=1}^{V} \sum_{\substack{S_{n}=1 \\
S_{n} \neq S_{n-1}, \cdots S_{1}}}^{n} \cdots \sum_{\substack{S_{2}=1 \\
S_{2} \neq S_{1}}}^{n} \sum_{S_{1}=1}^{n} \sum_{d=1}^{6} \sum_{t=1}^{T} x_{j v S_{n} \cdots S_{2} S_{1} d t}=1, \forall j \in \mathcal{J} \\
& \sum_{v=1}^{V} \sum_{S_{1}=1}^{n} \sum_{d=1}^{6} \sum_{t=1}^{T} x_{j v 0 \cdots 0 S_{1} d t} \leq 1, \forall j \in \mathcal{J}
\end{aligned}
$$




$$
\begin{aligned}
& \sum_{v=1}^{V} \sum_{S_{n}=1}^{n} \cdots \sum_{S_{2}=1}^{n} \sum_{S_{1}=1}^{n} \sum_{d=1}^{6} \sum_{t=1}^{T} x_{j v S_{n} \cdots S_{2} S_{1} d t} \leq 1, \forall j \in \mathcal{J} \\
& \sum_{d=1}^{6} \sum_{t=1}^{T}\left(x_{j v 0 \cdots 0 S_{1} d t}+\sum_{S_{k}=1}^{n} \cdots \sum_{\substack{\tilde{S}_{1}=1 \\
\tilde{S}_{1} \neq S_{1}}}^{n} x_{j v 0 \cdots 0 S_{k} \cdots \tilde{S}_{1} d t}\right) \leq 1, \\
& \forall k \in \mathcal{N} \backslash\{1\}, \forall j \in \mathcal{J}, \forall v \in \mathcal{V}, \forall S_{1} \in \mathcal{N} \\
& \sum_{S_{1}=1}^{n} \sum_{d=1}^{6} \sum_{t=1}^{T}\left(x_{j v 0 \cdots 0 S_{2} S_{1} d t}+\sum_{S_{k}=1}^{n} \cdots \sum_{\substack{\tilde{S}_{2}=1 \\
\tilde{S}_{2} \neq S_{2}}}^{n} x_{j v 0 \cdots 0 S_{k} \cdots \tilde{S}_{2} S_{1} d t}\right) \leq 1,
\end{aligned}
$$$$
\forall k \in \mathcal{N} \backslash\{1,2\}, \forall j \in \mathcal{J}, \forall v \in \mathcal{V}, \forall S_{2} \in \mathcal{N}(9)
$$

$$
\sum_{S_{n-2}=1}^{n} \cdots \sum_{S_{1}=1}^{n} \sum_{d=1}^{6} \sum_{t=1}^{T}\left(x_{j v 0 S_{n-1} \cdots S_{1} d t}+\sum_{\substack { S_{n}=1 \\
\begin{subarray}{c}{\tilde{S}_{n-1} \neq S_{n-1} \\
\tilde{S}_{n-1}{ S _ { n } = 1 \\
\begin{subarray} { c } { \tilde { S } _ { n - 1 } \neq S _ { n - 1 } \\
\tilde { S } _ { n - 1 } } }\end{subarray}}^{n} x_{j v S_{n} \tilde{S}_{n-1} S_{n-2} \cdots S_{1} d t}\right)
$$

$\sum_{S_{1}=1}^{n} \sum_{d=1}^{6} \sum_{t=1}^{T}\left(x_{j v 0 \cdots 0 S_{1} d t}+\sum_{\substack{\tilde{v}=1 \\ \tilde{v} \neq v}}^{V} \sum_{S_{2}=1}^{n} x_{j \tilde{v} 0 \cdots 0 S_{2} S_{1} d t}\right) \leq 1, \forall j \in \mathcal{J}, \forall v \in \mathcal{V}$

$\sum_{S_{1}=1}^{n} \sum_{d=1}^{6} \sum_{t=1}^{T}\left(x_{j v 0 \cdots 0 S_{1} d t}+\sum_{\substack{\tilde{v}=1 \\ \tilde{v} \neq v}}^{V} \sum_{S_{3}=1}^{n} \sum_{S_{2}=1}^{n} x_{j \tilde{v} 0 \cdots 0 S_{2} S_{1} d t}\right) \leq 1$,

$\sum_{S_{1}=1}^{n} \sum_{d=1}^{6} \sum_{t=1}^{T}\left(x_{j v 0 \cdots 0 S_{1} d t}+\sum_{\substack{\tilde{v}=1 \\ \tilde{v} \neq v}}^{V} \sum_{S_{n}=1}^{n} \cdots \sum_{S_{3}=1}^{n} \sum_{S_{2}=1}^{n} x_{j \tilde{v} 0 \cdots 0 S_{2} S_{1} d t}\right) \leq 1$,

$\forall j \in \mathcal{J}, \forall v \in \mathcal{V}$

$\sum_{\delta=1}^{\Delta} z_{j p m \delta}=\sum_{v=1}^{V} \sum_{S_{p}=1}^{n} \cdots \sum_{S_{1}=1}^{n} \sum_{d=1}^{6} \sum_{t \in T_{m}} P_{j v 0 \cdots 0 S_{p} \cdots S_{1} d t} x_{j v 0 \cdots 0 S_{p} \cdots S_{1} d t}$, 


$$
\begin{aligned}
& \tau_{j p}=\sum_{\delta=1}^{\Delta} \sum_{m=1}^{M} z_{j p m \delta}, \forall j \in \mathcal{J}, \forall p \in\left\{1, \ldots, n_{j}\right\} \\
& \frac{1}{2} \sum_{\delta=1}^{\Delta-1} \sum_{m=1}^{M} \delta\left|z_{j(p+1) m(\delta+1)}-z_{j(p+1) m \delta}\right|-\frac{\tau_{j(p+1)}}{2}+1 \\
& \quad \geq \frac{1}{2} \sum_{\delta=1}^{\Delta-1} \sum_{m=1}^{M} \delta\left|z_{j p m(\delta+1)}-z_{j p m \delta}\right|+\frac{\tau_{j p}}{2}, \forall j \in \mathcal{J}, \forall p \in\left\{1, \ldots, n_{j}-1\right\} \\
& \sum_{j=1}^{J} \sum_{p=1}^{n} z_{j p m \delta} \leq 1, \forall m \in \mathcal{M}, \forall \delta \in\{1, \ldots, \Delta\} \\
& \sum_{m=1}^{M} z_{j p m 1}+\sum_{m=1}^{M} z_{j p m \Delta}+\sum_{m=1}^{M} \sum_{\delta=1}^{\Delta-1}\left|z_{j p m(\delta+1)}-z_{j p m \delta}\right| \leq 2, \\
& \quad \forall j \in \mathcal{J}, \forall p \in\left\{1, \ldots, n_{j}\right\}
\end{aligned}
$$

\section{Case Study}

The proposed method was applied to a very simple example, since the total number of variables is generally huge. Two products $(J=2)$ of very simple shape (Fig. 5) for which two MPS sets $(V=2)$ with two MPSs $\left(n_{j}=2\right)$ are generated (Fig. 6) are produced by using two machines $(M=2)$. Three tools $(T=3)$ described in Table 1 was assumed. The length of a period was set to 400 [sec] to reduce the number of variables, and $\Delta$ was set to 40 . The calculation was carried out with a generic workstation (Dell Precision T7400, Intel Xeon E5430 2.66GHz, 20GB RAM) and the optimization was performed with a commercial solver (IBM ILOG CPLEX Optimization Studio 12.6). The calculation time was 3.9[sec]. The results by the conventional and proposed methods are shown in Tables 2 and 3, respectively. Shorter makespan could be achieved by the proposed method.

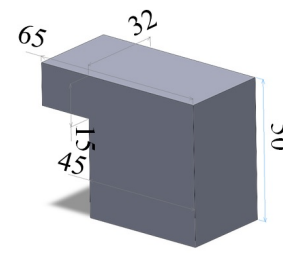

Product 1

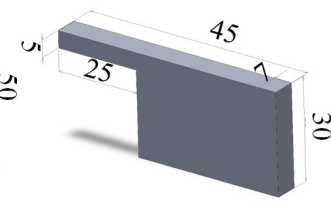

Product 2

Fig. 5. TRV of assumed products. The numbers show dimensions of them in $\mathrm{mm}$.
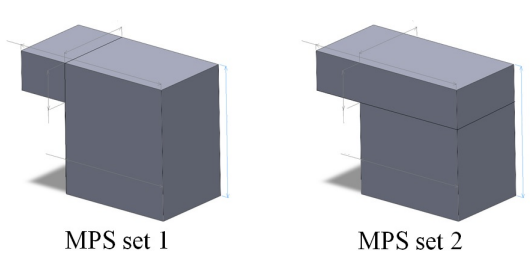

Fig. 6. MPS sets for product 1 . The numbers are the identification numbers for the MPSs. (MPS sets for product 2 are similar to these sets.) 
Table 1. Available tools.

\begin{tabular}{|c|c|c|c|c|c|}
\hline $\begin{array}{c}\text { Tool } \\
\text { ID }\end{array}$ & $\begin{array}{c}\text { Tool } \\
\text { Type }\end{array}$ & $\begin{array}{c}\text { Diameter } \\
{[\mathrm{mm}]}\end{array}$ & $\begin{array}{c}\text { The number of } \\
\text { cutting edge }\end{array}$ & $\begin{array}{c}\text { Breadth of cutting } \\
\text { edge }[\mathrm{mm}]\end{array}$ & $\begin{array}{c}\text { Machine } \\
\text { ID }\end{array}$ \\
\hline 1 & End mill & 4 & 2 & 12 & 1 \\
\hline 2 & End mill & 8 & 2 & 18 & 1 \\
\hline 3 & End mill & 10 & 4 & 21 & 2 \\
\hline
\end{tabular}

Table 2. Gantt chart obtained by the con- Table 3. Gantt chart obtained by the proventional method. posed method.

\begin{tabular}{|l|c|c|c|c|c|c|}
\hline Period & 1 & 2 & 3 & 4 & 5 & 6 \\
\hline Machine 1 & & & $2-2$ & & & \\
\hline Machine 2 & $2-1$ & $2-1$ & $1-1$ & $1-1$ & $1-2$ & $1-2$ \\
\hline
\end{tabular}

\begin{tabular}{|l|c|c|c|c|c|c|}
\hline Period & 1 & 2 & 3 & 4 & 5 & 6 \\
\hline Machine 1 & $2-1$ & $2-1$ & $2-1$ & $1-2$ & $1-2$ & \\
\hline Machine 2 & $1-1$ & $1-1$ & $1-1$ & $2-2$ & $2-2$ & \\
\hline
\end{tabular}

\section{Conclusion}

The conventional flexible CAPP method has been enhanced considering total productivity in production of multiple products. Selection of the optimal process plan in the conventional method and production scheduling were integrated and formulated as a 0-1 integer programming problem. This formulation was applied to a simple example and its potential was proven. The size of the integrated problem is generally huge and the proposed method cannot be applied to a real problem. This point will be discussed in a future work.

\section{Acknowledgement}

We thank MAZAK Foundation for its financial support.

\section{References}

1. Isnaini, M.M. and Shirase, K.: Review of Computer-Aided Process Planning Systems for Machining Operation - Future Development of a Computer-Aided Process Planning System - Int. J. Auto. Tech., 8, 317-332 (2014)

2. Sanchez, L.M. and Nagi, R.: A Review of Agile Manufacturing Systems, Int. J. Prod. Res., 39,3561-3600 (2001)

3. Nakamoto, K., Shirase, K., et al., Automatic Production Planning System to Achieve Flexible Direct Machining, JSME Int. J., Series C, 47, 136-143 (2004)

4. Hang, G., Koike, M., et al., Flexible Process Planning System Considering Design Intentions and Disturbance in Production Process, Mechatronics for Safety, Security and Dependability in a New Era, 113-118, Elsevier (2007)

5. Morinaga, E., Yamada, M., et al., Flexible Process Planning for Milling, Int. J. Auto. Tech., 5, 700-707 (2011)

6. Morinaga, E., Hara, T., et al., Improvement of Computational Efficiency in Flexible Computer-Aided Process Planning, Int. J. Auto. Tech., 8, 396-405 (2011)

7. Nagano, T., et al., Expert System Based on Case-Based Reasoning to Select Cutting Conditions (in Japanese), J. Japan Soc. Precision Eng., 67, 1485-1489 (2001) 